\title{
(2, 2)-Superconformal Field Theories near Orbifold Points
}

\author{
Paul Aspinwall*
}

Dept. Theoretical Physics, 1 Keble Rd, Oxford OX1 3NP, United Kingdom

\begin{abstract}
A thorough analysis of the "blowing-up" modes of the $\mathbb{Z}_{6}$-orbifold based on the Lie algebra $A_{2} \oplus D_{4}$ is presented. We discover that the descriptions of these modes in the language of superconformal field theory and Calabi-Yau compactification are not immediately in agreement. A solution to this apparent inconsistency is offered which leads to the possibility of differentiably distinct Calabi-Yau manifolds giving isomorphic physics.
\end{abstract}

\section{Introduction}

The fact that the superstring can propagate in ten-dimensional flat space-time led early models for superstring physics to be based on a ten-dimensional space globally of the form $M_{4} \times K$, where $K$ is a compact space of 6 dimensions. With this assumption, and many more, it was shown in [1] that, in order to have an unbroken $N=1$ space-time supersymmetry, $K$ must be a complex 3-fold with Kähler metric and vanishing Ricci-curvature. Manifolds admitting such a structure (i.e., Kähler manifolds with trivial canonical class) are known usually as Calabi-Yau manifolds. Superstrings can also propagate on compactification spaces with suitably mild singularities such as orbifolds [8] and therefore it seems appropriate to use a slightly wider class of spaces as candidates for $K$. In the following we will use $\mathrm{C}-\mathrm{Y}$ to denote this class of algebraic varieties possibly with singularities which may be blown up to give a smooth Calabi-Yau manifold.

A more general approach than the above ten-dimensional view is to take a superconformal field theory (SCFT) to model the compactification process. SCFTs are characterized by a central charge $\hat{c}$ giving operator products for the stress-energy tensor

$$
T_{B}\left(z_{1}\right) T_{B}\left(z_{2}\right) \sim \frac{\frac{3}{4} \hat{c}}{\left(z_{1}-z_{2}\right)^{4}}+\frac{2}{\left(z_{1}-z_{2}\right)^{2}} T_{B}\left(z_{2}\right)+\frac{1}{\left(z_{1}-z_{2}\right)} \partial_{2} T_{B}\left(z_{2}\right) .
$$

^ BITNET address: psa at ukacrl 
In order to cancel the ghost contribution to the central charge, we require a SCFT with $\hat{c}=6$ in order to obtain four-dimensional flat space-time. In this paper we will only consider the special case of the heterotic string with the spin connection identified with the gauge connection or the equivalent of this in conformal field theory language. For space-time supersymmetry in the heterotic string we require at least $(2,0)$ world-sheet symmetry with all physical vertex operators having integral $U(1)$ charge [the $U(1)$ associated to the $N=2$ supersymmetry] [2, 4]. Since we have identified the connection on the tangent bundle of the right moving superstring with the gauge connection on the bundle coming from the left movers, we will thus be considering (2,2)-superconformal field theories. Since a SCFT propagating on a circle $S^{1}$ has $\hat{c}=1$ (this is how $\hat{c}$ was normalized) we see that the product of six $\hat{c}=1$ theories satisfies our requirements (although we would actually get more supersymmetries than we want phenomenologically). Thus $\left(S^{1}\right)^{6}$ is a possible compactification space and since this is clearly a $\mathrm{C}-\mathrm{Y}$, we see agreement between the two theories. For general $\hat{c}=6$ SCFTs however, the connection with any $\mathrm{C}-\mathrm{Y}$ is far from apparent and we must use indirect methods to check for any equivalence between these theories. One approach [5] is to look for the spectrum of particles and discrete symmetries of the compactifications. We will follow the method initially considered in [6] and look at the moduli space of SCFTs which, if the theories are equivalent, should match the moduli space of C-Y's in some way. The moduli space of $c=1$ conformal field theories is, at least in part, well known [3]. The moduli space of $\hat{c}=1$ SCFTs has recently been studied in [7]. At generic points, the moduli space of any conformal field theory has the structure of a manifold where the number of dimensions is equal to the number of "truly marginal operators" $\Phi_{i}$. The field theory moves in this manifold by modifying the action

$$
S^{\prime}=S+g_{i} \int \Phi_{i} d z d \bar{z} .
$$

At certain points however ("multicritical points") truly marginal operators suddenly appear and we lose the manifold structure. In the simplest case, i.e., that of a $c=1$ conformal field theory connected to the circle we have a moduli space

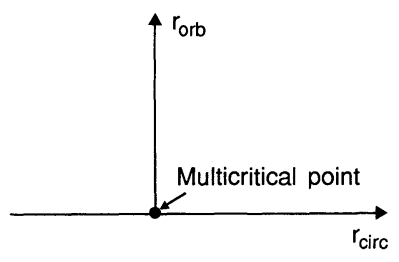

Seiberg [6] has already checked the agreement at smooth points of the moduli space. The moduli space of C-Y 3-folds is thought to have very interesting singular points however and it is a useful problem to look at the two types of theories around such points. The case of $\hat{c}=4$ has already been checked in [6]. Here, the only non-trivial $\mathrm{C}-\mathrm{Y}$ 's are $\mathrm{K} 3$ surfaces and since any $\mathrm{C}-\mathrm{Y}$ bimeromorphically equivalent to a $\mathrm{K} 3$ surface is biholomorphic to it we have no particularly interesting structure in the moduli space of $\mathrm{K} 3$ to look at. In the case of algebraic 3 -folds however, we have interesting birational geometry (see, for example [18]). 
The regions of the moduli space we will look at are the neighbourhoods of the points where a rational curve (holomorphic $\mathbb{P}^{1}$ ) has been blown-down. It is fortunate that it is at a set of points such as these that the only well-studied rigorous equivalence of SCFTs and C-Y's is known. These points are examples of orbifold models.

\section{Strings on Orbifolds}

An orbifold is a space in which the singularities are quotient singularities. One takes a smooth manifold $M_{0}$ which admits a discrete group $G_{0}$ which acts as isometries on $M_{0}$ and then one takes the quotient of $M_{0}$ by this group. - I.e., locally, in the neighbourhood of a fixed point, the space is homeomorphic to $\mathbb{R}^{n} / G$ for some discrete group $G$,

$$
g: x_{i} \mapsto\left(M_{g}\right)_{i j} x_{j}, \quad g \in G \leqq G_{0}
$$

for some matrix $M_{g}$,

and the points $\left(z_{i}\right)$ and $g\left(z_{i}\right)$ are identified. For a nontrivial group $G$, the origin of $\mathbb{R}^{n} / G$ will be singular since this is fixed by the action of $G$. Other points in $\mathbb{C}^{n}$ may also be fixed by some element and so these points will also project onto singular points in the orbifold. If, in a sufficiently small open neighbourhood, the origin is the only fixed point of any element of $G$, the orbifold is said to have an isolated singularity. In order to obtain an orbifold in the class $\mathrm{C}-\mathrm{Y}$, we need a space which will admit a holonomy group of $S U(3)$ and it can then be shown that we require $M_{0}$ to be a complex 3 -fold and that $G_{0} \subset S U(3)$.

Here we will obtain an orbifold in the following way [20]. Take the maximal torus $T_{\mathscr{L}}$ of a given Lie algebra $\mathscr{L}$. We then divide this torus by the cyclic group $G=\mathbb{Z}_{n}$ whose generator $g$ is the Coxeter element of $\mathscr{L}$. The Coxeter element is defined as the product of reflections about hyperplanes in the root lattice perpendicular to each simple root (i.e., the product of the generators of the Weyl group of $\mathscr{L}$ ). The Coxeter element is clearly an isometry of $T_{\mathscr{L}}$ and is a rotation for an even dimensional torus. Thus $\mathscr{L}$ fully defines an orbifold in this way. The action of the generator $g$ around a fixed point of $g$ is given by

$$
g:\left(z_{1}, z_{2}, z_{3}\right) \mapsto\left(e^{2 \pi i f_{1}} z_{1}, e^{2 \pi i f_{2}} z_{2}, e^{2 \pi i f_{3}} z_{3}\right),
$$

where the $f_{i}$ can be derived from $\mathscr{L}$. If we do not specify the fixed point to which the $f_{i}$ refer, the origin is assumed. The orbifold is flat everywhere that it is not singular. The well studied $Z$-orbifold [8] is given by $\mathscr{L}=A_{2} \oplus A_{2} \oplus A_{2}$. The only other sixdimensional orbifold from this class with only isolated singularities is the " $\mathbb{Z}_{7}$-orbifold" $\mathscr{L}=A_{6}$, where we have 7 singularities locally of the form $\mathbb{C}^{3} / \mathbb{Z}_{7}$, $f=\frac{1}{7}(1,2,4)$. Unfortunately these two orbifolds do not exhibit the properties we are interested in and we shall study another orbifold based on $A_{2} \oplus D_{4}$. This has values $f_{i}=\frac{1}{6}(1,2,3)$ where the middle co-ordinate here refers to the $A_{2}$ part and the outer two co-ordinates are associated to $D_{4}$. We can see immediately that the cube of the Coxeter generator acts as $\frac{1}{2}(1,0,1)$, thus fixing the whole $A_{2}$ torus and we do not 
therefore have just isolated singularities. The fixed point sets are as follows:

$$
\begin{array}{ll}
g: \frac{1}{6}(1,2,3) \text { has } 12 \text { fixed points: } & 3 \text { on } A_{2} \\
& 4 \text { on } D_{4}, \\
g^{2}: \frac{1}{3}(1,2,0) \text { has } 9 \text { fixed tori: } & 3 \text { fixed points on } A_{2} \\
& 3 \text { fixed tori on } D_{4}, \\
g^{3}: \frac{1}{2}(1,0,1) \text { has } 16 \text { fixed tori: } & A_{2} \text { is fixed } \\
& 16 \text { fixed points on } D_{4},
\end{array}
$$

which leads to the configuration on $T_{\mathscr{L}}$ shown in Fig. 1. The tori (A) are mapped to themselves trivially by $\mathbb{Z}_{2} \subset \mathbb{Z}_{6}$ and to each other in groups of threes by $\mathbb{Z}_{3}$. The tori (B) are mapped to themselves trivially by $\mathbb{Z}_{2}$ and non-trivially by $\mathbb{Z}_{3}$. (C) are mapped to themselves non-trivially by $\mathbb{Z}_{2}$ and trivially by $\mathbb{Z}_{3}$ and (D) are mapped to each other in pairs by $\mathbb{Z}_{2}$ and to themselves trivially by $\mathbb{Z}_{3}$. The twelve points of intersection of the tori (B) and (C) are the only points mapped to themselves by all the elements of $\mathbb{Z}_{6}$.

In order to describe the strings that propagate on this orbifold we will use the language of conformal field theory. We begin with the basic $\hat{c}=6$ SCFT, where the fields $X^{\mu}$ simply describe the torus $T_{\mathscr{L}}$. We then "mod out" by the group $G$ in a well studied way to obtain another $\hat{c}=6$ SCFT $[10,11]$. This new SCFT consists of states invariant under $G$ from the original torus but we also obtain new "twisted" states demanded by one-loop modular invariance of the theory.

A physical string is described by its vertex operator - namely a primary field of conformal dimension $(h, \bar{h})=(1,1)$. The right moving sector of the heterotic string is that of a superstring with (at least) $N=1$ world-sheet supersymmetry. The right-
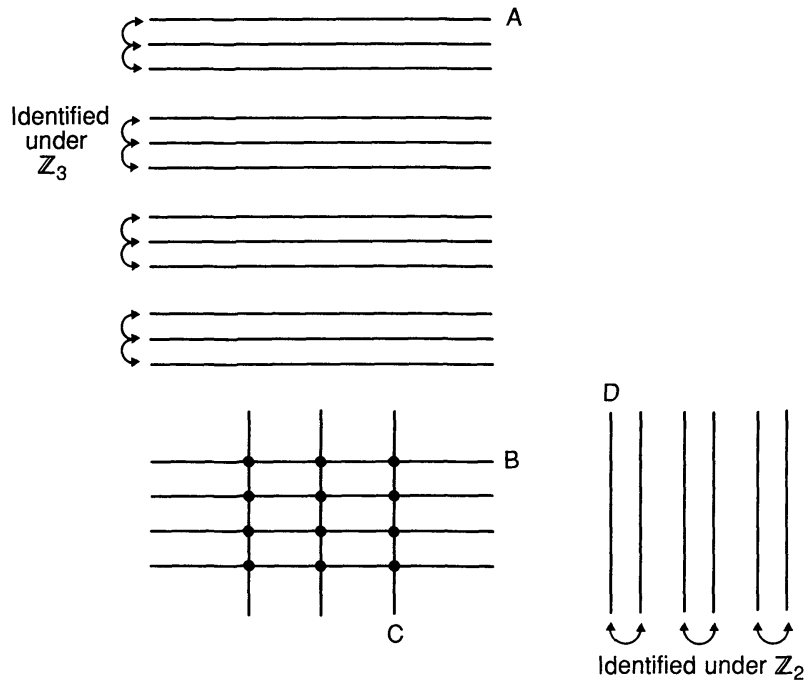

Fig. 1. The configuration of fixed points on the torus $T_{\mathscr{L}}$ 
handed part of the vertex operator for the emission of untwisted bosons and fermions in the $(-1)$ and $\left(-\frac{1}{2}\right)$ pictures respectively are [9]

$$
\begin{gathered}
V_{-1}(\text { boson })=e^{i \alpha_{b} \cdot H} e^{i k X} e^{-\phi}, \\
V_{-1 / 2}(\text { fermion })=e^{i \alpha_{f} \cdot H} e^{i k X} e^{-1 / 2 \phi} .
\end{gathered}
$$

Here the NSR fermionic degrees of freedom have been bosonized into five degrees of freedom $H^{i},(i=1 \ldots 5)$. The $\alpha^{i}$ give the momenta on the weight lattice of $S O(10)$ corresponding to these degrees of freedom. Given that the conformal weight of $e^{l \phi}$ is $-\frac{1}{2} l(l+2)$, for $h=1$ we have

$$
\begin{aligned}
& \frac{1}{2} \alpha_{b}^{2}-\frac{1}{2} k^{2}+\frac{1}{2}=1, \\
& \frac{1}{2} \alpha_{f}^{2}-\frac{1}{2} k^{2}+\frac{3}{8}=1,
\end{aligned}
$$

so that for massless bosons we have $\alpha_{b}=( \pm 1,0,0,0,0), \ldots$ i.e., the 10 of $S O(10)$ and for massless fermions $\alpha_{f}=\left( \pm \frac{1}{2}, \pm \frac{1}{2}, \ldots\right)$ (even number of + signs) i.e., the 16 of $S O(10)$ showing we have a vector and a spinor respectively. For the twisted sectors we introduce a twist operator $\Lambda_{g}$ which takes us from an untwisted state to a twisted one. The conformal weight of $\Lambda_{g}$ can be shown to be $h_{\Lambda}=\frac{1}{2} \sum_{i=1}^{3} f_{i}\left(1-f_{i}\right)$. Our boson vertex now becomes

$$
V_{-1}(\text { twisted boson })=e^{i \alpha_{b} \cdot H} \Lambda_{g} e^{i k X} e^{-\phi} .
$$

The twist results in a change in boundary conditions for the NSR fermions and so our $S O(10)$ lattice must be affected. An $S U(3) \subset S O(6)$ element $g=\operatorname{diag}\left(e^{2 \pi i f_{1}}, e^{2 \pi i f_{2}}, e^{2 \pi i f_{3}}\right)$ can be represented as a translation on the maximal torus of $D_{3}$ of $\left(f_{1}, f_{2}, 1-f_{3}\right)$, where we have used co-ordinates such that the simple roots of $D_{3}$ are $(1,-1,0),(0,1,1)$, and $(0,1,-1)$. This embeds naturally as the first three co-ordinates in the root space of $D_{5}$, the algebra of $S O(10)$ and, in fact, it is this translation which acts on our torus for the NSR fermions.

As an example, consider the twist $\frac{1}{6}(1,2,3)$. This will shift the lattice by an amount $v=\left(\frac{1}{6}, \frac{1}{3}, \frac{1}{2}, 0,0\right)$. For a vector $p$ on the unshifted lattice we thus have

$$
\frac{1}{2}(p-v)^{2}+\frac{1}{2} \times\left(\frac{1}{6} \times \frac{5}{6}+\frac{1}{3} \times \frac{2}{3}+\frac{1}{2} \times \frac{1}{2}\right)-\frac{1}{2} k^{2}+\frac{1}{2}=1
$$

satisfied, for $k=0$, by $p=(0,0,1,0,0)$ giving a scalar. Similarly for the fermion we have

$$
\frac{1}{2}(p-v)^{2}+\frac{11}{36}+\frac{3}{8}=1
$$

satisfied by $p=\left(\frac{1}{2}, \frac{1}{2}, \frac{1}{2}, \pm \frac{1}{2}, \mp \frac{1}{2}\right)$ giving a chiral fermion in four dimensions. Notice that a shift of $-v$ will give us the opposite chirality. In the left moving sector we have no superghost charges and the $S O(10)$ lattice is replaced by an $E_{8} \times E_{8}$ lattice. We will use only one $E_{8}$ as the second contributes nothing of importance here. The $S O(6)$ lattice naturally embeds into the $E_{8}$ lattice and so, for $(2,2)$-models, a twist results in a shift of $\left(f_{1}, f_{2}, 1-f_{3}, 0,0,0,0,0\right)$. (We have used here the usual basis for the $E_{8}$ lattice - see [8].) The twisted sector then has vertex operators such as

$$
V_{\mathbf{2 7}}=e^{i \tilde{\alpha} \cdot F} \Lambda e^{i k X} \text {. }
$$

For twists such as $\frac{1}{6}(1,2,3)$, this leads to massless states forming a 27 of $E_{6} \subset E_{8}$. Note that the correspond anti-twist sector gives us a $\overline{27}$ representation. We can 
also obtain massless states using oscillator modes of the form

$$
V_{m}=e^{i \tilde{\alpha} \cdot F} \Lambda \frac{\partial X}{\partial \bar{z}^{f_{\imath}}} e^{i k X}
$$

Let us look at a twist with a fixed line through the origin rather than an isolated fixed point e.g., $\frac{1}{3}(1,2,0)$. It is best to treat this as an $S U(2)$ element and embed it in the so (4) algebra and represent $v$ by $\left(\frac{1}{3}, \frac{1}{3}, 0,0, \ldots\right)$. For the massless fermions we now get

$$
\frac{1}{2}(p-v)^{2}+\frac{2}{9}+\frac{3}{8}=1
$$

satisfied by $p=\left(\frac{1}{2}, \frac{1}{2}, \pm \frac{1}{2}, \pm \frac{1}{2}, \pm \frac{1}{2}\right)$ (even number of plus signs) and so we have both chiralities of each fermion present in four dimensions.

In the left sector we now have

$$
\frac{1}{2}(\tilde{p}-v)^{2}+\frac{2}{9}=1,
$$

which gives us $\tilde{p}=(1,0, \ldots, \pm 1, \ldots),(0,1, \ldots, \pm 1, \ldots)$ or $\left(\frac{1}{2}, \frac{1}{2}, \pm \frac{1}{2}, \pm \frac{1}{2}, \ldots\right)$ (with an even number of + signs). These weights form the 56 of $E_{7} C E_{8}$. We are looking for $E_{6}$ representations and so we obtain both a 27 and a $\overline{27}$ from this sector. We have therefore a particle + anti-particle pair of 27 and a particle + anti-particle pair of $\overline{27}$. In the isolated fixed point case we obtained only a particle of a 27 representation. One can understand from this the significance of compactification on $\mathrm{K} 3 \times$ torus - an example of a $\mathrm{C}-\mathrm{Y}$. This has Euler characteristic $=0$ and so we should not expect to have any net number of chiral generations. We can build K3 $\times$ torus from an orbifold with only non-isolated fixed points similar (or indeed precisely the same) as those above and we thus automatically pair off every 27 with a $\overline{27}$.

We are now in a position to piece together the above information to build the spectrum of $27 \mathrm{~s}$ and $\overline{27} \mathrm{~s}$ appearing in the $A_{2} \oplus D_{4}$ orbifold. The sectors twisted by $g$ and $g^{5}$ are easy - these are just like the twisted sectors of the $Z$-orbifold [8]. For each of the twelve fixed points of $g$ we obtain a 27, the $g$ and $g^{5}$ giving the particle and anti-particle respectively. For the other twisted sectors we mustn't forget that, like untwisted strings, it is necessary to project onto the Hilbert space of $g$ invariant states. To do this we require that for a sector twisted by $t=g^{m}$, our twisted strings are invariant under the quotient group $G / T \simeq \mathbb{Z}_{\frac{m}{n}}^{n}$, where $n$ is the order of $g$ and $T$ is the cyclic group generated by $t$. Thus, turning to the sector twisted by $g^{2}$ we require that such states should be invariant under the $\mathbb{Z}_{2}$ symmetry generated by $g^{3}$. For each of the tori(D), fixed by $g^{2}$ and $g^{4}$ we have a local action of $g^{2}$ of $\frac{1}{3}(1,2,0)$ and so from the above reasoning we obtain both a 27 and a $\overline{27}$ representation. We also obtain a second 27 and $\overline{\mathbf{2 7}}$ from the $g^{4}$-twisted sector. The $\mathbb{Z}_{2}$ identifies the (D) tori in pairs thus halving the above number of massless particles giving $6 \times 27$ and $6 \times \overline{\mathbf{2 7}}$ from here. A similar analysis of the (A) tori gives another $4 \times \mathbf{2 7}$ and $4 \times \overline{\mathbf{2 7}}$. The $(\mathrm{C})$ tori, like the $(\mathrm{D})$ tori, begin life before the projection onto $g$-invariant states with two $27 \mathrm{~s}$ and $\overline{27} \mathrm{~s}$ each but here the $\mathbb{Z}_{2}$ acts within each sector acting as the identity on the 27 s but as -1 on the $\overline{27}$ s so that the $\overline{27}$ s are projected out leaving us with 627 s from the $(C)$ tori. The $(B)$ tori similarly give $427 \mathrm{~s}$. We now have a grand total of $3227 \mathrm{~s}$ and $10 \overline{27} \mathrm{~s}$ coming from the twisted sectors which when added to the untwisted states gives $35 \mathrm{27}$ s and $11 \overline{27} \mathrm{~s}$, giving a net number of 24 generations which may be seen to be in agreement with the index formula from [8]. 
What we are interested in are the truly marginal operators of the SCFT based on this orbifold. It has been shown $[2,12]$ that the world-sheet supersymmetry of a $(2,2)-S C F T$ may be used to establish a one-to-one correspondence between 27 and 27 matter fields like those above. The marginal operators preserving the world sheet supersymmetry have the form

so that

$$
\Phi^{ \pm, \pm}=\Phi_{0,0}^{ \pm, \pm}+\bar{\theta} \Phi_{1,0}^{ \pm, \pm}+\theta \Phi_{0,1}^{ \pm, \pm}+\bar{\theta} \theta \Phi_{1,1}^{ \pm, \pm}
$$

$$
S^{\prime}=S+g_{i} \int \Phi_{i} d \theta d \bar{\theta} d z d \bar{z}
$$

The vertex operators of the fields $\Phi_{0,0}^{-} \pm$can be written [6]

$$
V_{I}=\varepsilon_{k}^{\overline{i j}} f_{\overline{i j} l}^{I} \bar{\psi}^{l} \bar{\psi}^{k}
$$

where $I$ labels the cohomology class of the $(2,1)$-forms and the fields $\Phi_{0,0}^{+}{ }^{ \pm}$are

where $J$ labels $(1,1)$-forms.

$$
V_{J}=b_{i i}^{J} \bar{\psi}^{\bar{i}} \psi^{i}
$$

From the fields $\Phi_{0,1}^{+, \pm}\left(\Phi_{0,1}^{-, \pm}\right)$we can also obtain the vertex operators for the $27 \mathrm{~s}$ $(\overline{27} \mathrm{~s})$ thus giving a one-to-one correspondence. For the truth of the marginal operators we refer to $[2,12]$.

\section{Geometry of Blow-ups}

We now look at the orbifold model from the perspective of compactification on a $\mathrm{C}-\mathrm{Y}$. After all, an orbifold is just a particular point in the moduli space of a $\mathrm{C}-\mathrm{Y}$ and we should be able to move continuously to a smooth model by resolving the orbifold singularities. As we shall see, this process is not always unique and this raises some interesting questions for superstring compactification.

We can cover any complex orbifold by co-ordinate patches which are biholomorphic to either $\mathbb{C}^{3}$ or $\mathbb{C}^{3} / G$. We will resolve the singularities in the orbifold by applying a bimeromorphic transformation to $\mathbb{C}^{3} / G$ to obtain a manifold with the same asymptotic topology away from the origin as $\mathbb{C}^{3} / G$. We can then cover the whole space with $\mathbb{C}^{3}$ co-ordinate patches and thus have a complex 3-fold. In order for this resultant manifold to be a Calabi-Yau manifold so that we might expect it to be a superstring compactification candidate, we must satisfy the requirements of being Kähler and having trivial canonical bundle, $K$. (The condition $K=0$ is equivalent to the vanishing of the first Chern class of the tangent bundle of the manifold.) As we shall see, these requirements are needed to render the number of possible resolutions potentially finite.

To begin our discussion of resolutions let us review the "monoidal transformation" or blow-up of a non-singular point. Over a complex projective space $\mathbb{P}^{n}$ we have a natural line bundle $L=\mathcal{O}_{\mathbb{P}^{n}}(-1)$. We will use the notation $\mathcal{O}_{\mathbb{P}^{n}}(k), k \in \mathbb{Z}$ for the $k^{\text {th }}$ power of the hyperplane bundle over $\mathbb{P}^{n}$. The hyperplane bundle is the line bundle $H \rightarrow \mathbb{P}^{n}$ which admits global holomorphic sections with a zero-locus corresponding to a hyperplane $\mathbb{P}^{n-1}$ of $\mathbb{P}^{n}$. The total space of the line bundle $\mathcal{O}(-1)$ may be considered as a submanifold of $\mathbb{C}^{n+1} \times \mathbb{P}^{n}$

$$
L \sim\left\{(z, l): z_{i} l_{j}=z_{j} l_{i}\right\},
$$


where

$$
\left(z_{0}, \ldots, z_{n}\right) \in \mathbb{C}^{n+1}, \quad\left[l_{0}, \ldots, l_{n}\right] \in \mathbb{P}^{n} .
$$

This space, $\mathbb{C}^{n+1}$, is easily seen to be isomorphic to $\mathbb{C}^{n+1}$ away from the origin $z=(0, \ldots)$. At the origin however we have a $\mathbb{P}^{n}$. The origin of $\mathbb{C}^{n+1}$ is said to have been "blown-up" at the origin or a "monoidal transformation" has been applied at the origin to obtain $\widetilde{\mathbb{C}}^{n+1}$. This new $\mathbb{P}^{n}$ replacing the point at the origin is called the exceptional divisor of the blow-up. Considering the exceptional divisor as a submanifold of co-dimension 1, we can clearly have a normal line bundle over this Weyl divisor of $\mathcal{O}_{\mathbb{P}^{n}}(-1)$ and indeed any such submanifold of a complex manifold can be blown-down back to a non-singular point. For a manifold $M$ blown-up to $\tilde{M}$ it is usual to use the notation

$$
\pi: \tilde{M} \rightarrow M,
$$

where $\pi$ is the blowing-down procedure. It is not difficult to show that, for an exceptional divisor $E$ in the case of a monoidal transformation

$$
K_{\tilde{M}}=\pi^{*} K_{M}+n E \text {. }
$$

(We will be somewhat sloppy when distinguishing between line bundles and divisors.) Thus, if $n>0, K_{\tilde{M}}$ cannot be trivial. This is indeed fortunate as otherwise any $\mathrm{C}-\mathrm{Y}$ could be blown-up anywhere any number of times giving a very infinite number of superstring compactifications.

Another point we need to look at here is the subject of the projectivity of a monoidal transformation. A manifold $\tilde{M}$ is projective if we can construct a very ample line bundle on $\tilde{M}$. Equivalently we can think of this as constructing a positive $(1,1)$-form to act as the Kähler form on $\tilde{M}$. It can be shown [13] that given a very ample line bundle $L$ on $M$ we can construct another very ample bundle $\widetilde{L} \rightarrow \tilde{M}:$

$$
\widetilde{L}=\pi^{*} L^{k}-E,
$$

where $k$ is a sufficiently large integer. Thus we start with a Kähler manifold and blow-up at a point and obtain a new Kähler manifold with part of the new Kähler form associated with the exceptional divisor. By varying the magnitude of this part of the Kähler form we change the volume of the exceptional divisor since we can define the volume by

$$
\operatorname{Vol}(E)=\int_{E} J^{n}
$$

where $J$ is the Kähler form on the $(n+1)$-fold $\tilde{M}$. This deformation of $J$ is therefore sometimes referred to as the "blow-up mode" of deformations of $\tilde{M}$ and is associated with the cohomology class $H_{\partial}^{1}, 1(\tilde{M})$ dual to the exceptional divisor.

Now, consider what happens if we take not $\mathcal{O}_{\mathbb{P} n}(-1)$ for the normal bundle of our submanifolds, but $\mathcal{O}_{\mathbb{P}^{n}}(-k)$. In $\mathcal{O}(-1)$ we have co-ordinate patches

$$
\begin{aligned}
& U_{0}\left(l_{0} \neq 0\right): \xi_{0}=\frac{l_{1}}{l_{0}}, y_{0}=z_{0}, \\
& U_{1}\left(l_{1} \neq 0\right): \xi_{1}=\xi_{0}^{-1}, y_{1}=y_{0} \xi_{0} .
\end{aligned}
$$


Thus, for $\mathcal{O}(-k)$ we have

$$
\begin{aligned}
& U_{0}: \xi_{0}, y_{0} \\
& U_{1}: \xi_{1}=\xi_{0}^{-1}, y_{1}=y_{0} \xi_{0}^{k} .
\end{aligned}
$$

The transition functions are therefore as if $\left(z_{0}, \ldots, z_{n}\right)$ have been replaced by $\left(z_{0}^{k}, \ldots, z_{n}^{k}\right)$ and so if we consider the projection

$$
p: \mathbb{C}^{n+1} \backslash\{0\} \rightarrow \mathcal{O}(-k) \backslash\{z=0\} .
$$

We now have the identification

$$
p\left(z_{0}, \ldots, z_{n}\right) \simeq p\left(e^{\frac{2 \pi i}{k}} z_{0}, \ldots, e^{\frac{2 \pi i}{k}} z_{n}\right),
$$

and so, away from the origin, this space is isomorphic to $\mathbb{C}^{n+1} / \mathbb{Z}_{k}$ where, in the notation of the previous section, the generator of $\mathbb{Z}_{k}$ acts as $\frac{1}{k}(1,1, \ldots)$. This is then precisely what we require as a resolution of such a quotient singularity. Blowing down the exceptional divisor will leave a quotient singularity at the origin. On the singular manifold $M$, we can suitably define a Kähler form and canonical class (but these will degenerate at the singular origin). For the canonical class of $\tilde{M}$, we can generalize (22) to obtain

$$
K_{\tilde{M}}=\pi^{*} K_{M}+(n-k+1) E .
$$

If we want to obtain a $\mathrm{C}-\mathrm{Y}$ we therefore require

$$
k=n+1 \text {. }
$$

In the case of complex surfaces, the resolution of quotient singularities is well studied. For a thorough review, see [14]. Here $n=1$ so we can resolve $\mathbb{C}^{2} / \mathbb{Z}_{2}$ with $\mathcal{O}_{\mathbb{P}^{1}}(-2)$ (the space which admits an Eguchi-Hanson metric [15]) as is well known from the usual construction of K3. What about other possible Ricci-flat blow-ups? To obtain $S U(2)$ holonomy we require, for a quotient $\mathbb{C}^{2} / G$, that $G \subset S U(2)$. The resolution now consists of a series of $\mathcal{O}_{\mathbb{P} 1}(-2)$ blow-ups. Each blow-up can leave a quotient singularity on the exceptional curve and we continue blowing-up until the space is everywhere smooth. We thus obtain a total exceptional divisor of $\mathbb{P}^{1}$ 's touching at points. It can be shown that the resultant configuration of curves correspond to Dynkin diagrams of simply laced Lie algebras giving the most beautiful correspondence between simply laced Lie algebras and discrete subgroups of $S U(2)$.

For the case of threefolds, the subject we are interested in, the situation is much less pleasant. It is simple to see that the line bundle $\mathcal{O}_{\mathbb{P}^{2}}(-3)$ gives the resolution of $\mathbb{C}^{3} / \mathbb{Z}_{3}$ which can thus be used for the case of the $Z$-orbifold. Any other $G \subset S U(3)$ will require additional tools.

The extra machinery we will use is the geometry of toric varieties. For a proof of all the statements concerning toric varieties that follow, the reader is referred to [16]. Toric resolutions have also recently been studied in connection with CalabiYau manifolds in [20,21]. We begin with two free modules $M, N \cong \mathbb{Z}^{r}$ with $M$ and 
Fig. 2. $A$ fan in $\mathbb{R}^{2}$

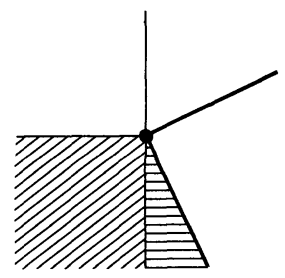

$N$ dual with respect to some product

$$
\langle,\rangle: M \times N \rightarrow \mathbb{Z} .
$$

We also fill in these lattices to form the spaces

$$
N_{\mathbb{R}}=N \otimes_{\mathbb{Z}} \mathbb{R}, \quad M_{\mathbb{R}}=M \otimes_{\mathbb{Z}} \mathbb{R} .
$$

By a cone, $\sigma$, we will mean a subset of $N_{\mathbb{R}}$ given by $n_{1}, n_{2}, \ldots, n_{s} \in N$ such that

$$
\begin{aligned}
\sigma & =\mathbb{R}_{+} n_{1}+\ldots+\mathbb{R}_{+} n_{s} \\
& =\left\{a_{1} n_{1}+\ldots+a_{s} n_{s} ; a_{i} \in \mathbb{R}, a_{i} \geqq 0, i=1 \ldots s\right\}
\end{aligned}
$$

and the space is convex. We thus have some convex polyhedral based cone with its apex at the origin. Clearly, faces of this cone are also cones. We make fans, $\Sigma$, by sticking together cones, see Fig. 2. For our purposes we will actually only be interested in fans where each cone is, or is a face of some cone containing $r$ dimensional space. Figure 2 is thus excluded because of the two isolated lines. We can now define a complex $r$-fold, $X_{\Sigma}$, associated to a fan $\Sigma$ in $\mathbb{R}^{r}$ by the following recipe:

1. Build a set of cones in $M_{\mathbb{R}}$. To each cone $\sigma$ in $N_{\mathbb{R}}$ we define $\sigma^{\vee}$ in $M_{\mathbb{R}}$ by

$$
\sigma^{\vee}=\left\{x \in M_{\mathbb{R}} ;\langle x, y\rangle \geqq 0 \forall y \in \sigma\right\} .
$$

2. In every cone containing $r$-dimensional space, $\sigma_{i}^{\vee}$, we determine the minimum set of elements $\left\{m_{i j} \in M\right\}, j=1 \ldots p, p \geqq r$ such that

$$
M \cap \sigma_{i}^{\vee}=m_{i 1} \mathbb{Z}_{+}+m_{i 2} \mathbb{Z}_{+}+\ldots+m_{i p} \mathbb{Z}_{+} .
$$

3. Associate to this cone a space $U_{\sigma_{i}}$

$$
U_{\sigma_{i}}=\left\{\left(u_{i 1}, \ldots, u_{i p}\right) \in \mathbb{C}^{p} ; u_{i 1}^{h_{1}^{\alpha}} u_{i 2}^{h_{2}^{\alpha}} \ldots=1, \alpha=1 \ldots p-r\right\},
$$

where the $h_{j}^{\alpha} \in \mathbb{Z}$ are obtained from the maximal set of independent relations

$$
h_{1}^{1} m_{i 1}+h_{2}^{1} m_{i 2}+\ldots+h_{p}^{1} m_{i p}=0 \text {. }
$$

4. Glue together the co-ordinate charts $U_{\sigma_{i}}$ with the rule that relationships like

$$
l_{i 1} m_{i 1}+l_{i 2} m_{i 2}+\ldots+l_{j 1} m_{j 1}+l_{j 2} m_{j 2}+\ldots=0, \quad l_{i p} \in \mathbb{Z}
$$

go to

$$
u_{i 1}^{l_{i 1}} u_{i 2}^{l_{i 2}} \ldots u_{j 1}^{l_{j 1}} u_{j 2}^{l_{j 2}} \ldots=1
$$




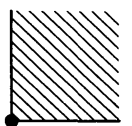

$\mathbb{C}^{2}$

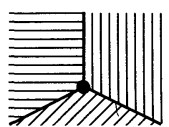

$\mathbb{P}^{2}$

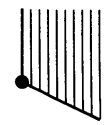

$\mathbb{C}^{2} / \mathbb{Z}_{2}$

Fig. 3. Examples of fans representing toric surfaces

We then have the following theorems which will prove useful. Define the minimal set $\left\{n_{i j}\right\}$ similarly to $m_{i j}$,

then we have

$$
N \cap \sigma_{i}=n_{i 1} \mathbb{Z}_{+}+\ldots+n_{i q} \mathbb{Z}_{+},
$$

Theorem 1. $X_{\Sigma}$ is smooth if and only if, for every $\sigma_{i}$ enclosing a space of dimension $r$,

i.e., $q=r$.

$$
\sigma_{i}=\mathbb{R}_{+} n_{i 1}+\ldots+\mathbb{R}_{+} n_{i r},
$$

Theorem 2. $X_{\Sigma}$ is compact if and only if the fan $\Sigma$ covers the whole of $N_{\mathbb{R}}$.

The examples of Fig. 3 should prove helpful in understanding how to build $X_{\Sigma}$ and illustrating the above theorems.

We are interested in smoothing out singularities to obtain Calabi-Yau manifolds and hence require

Theorem 3. If the conditions for Theorem 1 are satisfied then

$$
K_{X_{\Sigma}}=0 \Leftrightarrow \begin{aligned}
& \left\{n_{i j}\right\} \text { lie in a hyperplane } \\
& H_{N} \text { of } \mathbb{R}^{r} .
\end{aligned}
$$

Thus we can see that a compact toric variety cannot have trivial canonical bundle. Since, for our purposes $r=3$, we have $H_{N} \simeq \mathbb{R}^{2}$ and we can draw the diagram representing $\Sigma$, if $K_{X_{\Sigma}}=0$, by drawing the $\left\{n_{i j}\right\}$ on the plane of our piece of paper - i.e., draw the section through $\Sigma$ containing $\left\{n_{i j}\right\}$.

So, can we represent our singular space $\mathbb{C}^{n} / G$ as a toric variety? The answer is yes so long as $G$ is abelian and can thus be diagonalized into the form (4). In order to avoid a lengthy exposition of toric geometry, we will again just give a recipe for constructing our fan $\Sigma_{f}$ from $\left(f_{1}, f_{2}, f_{3}\right)$. The fan $\Sigma_{f}$ corresponding to $\left(f_{1}, f_{2}, f_{3}\right)$ $=\frac{1}{n}\left(1, a_{2}, a_{3}\right)$, where $a_{i}, n \in \mathbb{Z}$ may be given by the cone $\sigma_{f}$ and its faces,

$$
\sigma_{f}=\mathbb{R}_{+} \kappa_{1}+\mathbb{R}_{+} \kappa_{2}+\mathbb{R}_{+} \kappa_{3},
$$

where

$$
\kappa_{1}=\left(\begin{array}{c}
n \\
-a_{2} \\
-a_{3}
\end{array}\right), \quad \kappa_{2}=\left(\begin{array}{l}
0 \\
1 \\
0
\end{array}\right), \quad \kappa_{3}=\left(\begin{array}{l}
0 \\
0 \\
1
\end{array}\right),
$$



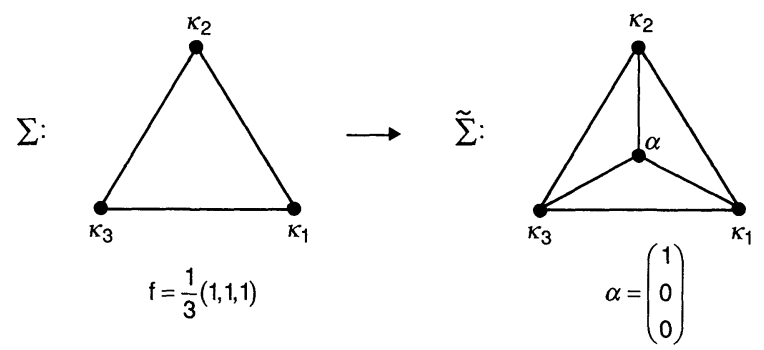

Fig. 4. Resolution of the quotient singularity in the $Z$-orbifold

Now, given a triangular cone such as the one above defined by the three lattice vectors $\alpha_{i}, i=1 \ldots 3$, we can see that, to satisfy Theorem 1 for a smooth space, we require $\operatorname{det}(\alpha)=1$. Since $\operatorname{det}(\kappa)=n$, we have a singular variety for $n>1$ as we should expect. Let us consider the familiar singularity from the $Z$-orbifold $f=\frac{1}{3}(1,1,1)$ thus

$$
\kappa_{1}=\left(\begin{array}{c}
3 \\
-1 \\
-1
\end{array}\right), \quad \kappa_{2}=\left(\begin{array}{l}
0 \\
1 \\
0
\end{array}\right), \quad \kappa_{3}=\left(\begin{array}{l}
0 \\
0 \\
1
\end{array}\right) \text {. }
$$

In order to satisfy Theorems 1 and 3, it seems natural to subdivide this cone to obtain a non-singular (in the sense of Theorem 1) fan as shown in Fig. 4. Following the recipe for building $X_{\Sigma}$, one may verify that, as one would hope, $X_{\tilde{\Sigma}}$ is indeed the space of the line bundle $\mathcal{O}_{\mathbb{P}^{2}}(-3)$. This then is blowing up in the language of toric varieties - we subdivide out cone until we have a fan satisfying Theorems 1 and 3. It should be noted that once these conditions are satisfied, no further subdivision of the fan is possible without violating Theorem 3 . When building $\mathcal{O}_{\mathbb{P}^{2}}(-3)$ from the co-ordinate patches from $\tilde{\Sigma}$, one can see that the exceptional divisor is associated with the cone $\mathbb{R}_{+} \alpha$. This is a general feature of toric varieties - subcones of dimension $s$ in $\Sigma$ are associated with submanifolds of dimension $r-s$ in $X_{\Sigma}$.

Before continuing into resolving the singularities of our orbifold we need to study some aspects of rational curves. Any holomorphic vector bundle over $\mathbb{P}^{1}$ is isomorphic to a sum of line bundles over $\mathbb{P}^{1}$. Thus, any rational algebraic curve in a complex 3-fold has associated to it, for the normal bundle of the curve $\mathcal{N}_{\mathbb{P}^{1} / X}$, two integers $a_{1}$ and $a_{2}$ such that

$$
\mathscr{N}_{\mathbb{P}^{1} / X} \simeq \mathcal{O}_{\mathbb{P}^{1}}\left(a_{1}\right) \oplus \mathcal{O}_{\mathbb{P}^{1}}\left(a_{2}\right) .
$$

Applying the adjunction formula, for a rational curve in a variety with $K=0$, we have

$$
a_{1}+a_{2}=-2 \text {. }
$$

We shall refer to such a curve as a $\left(a_{1}, a_{2}\right)$-curve. In out toric varieties, curves will appear as 2-cones and thus as lines in our diagrams of $\Sigma \cap H$. It can be shown that, for a sub-fan

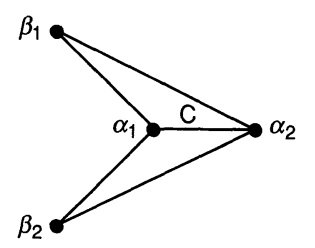



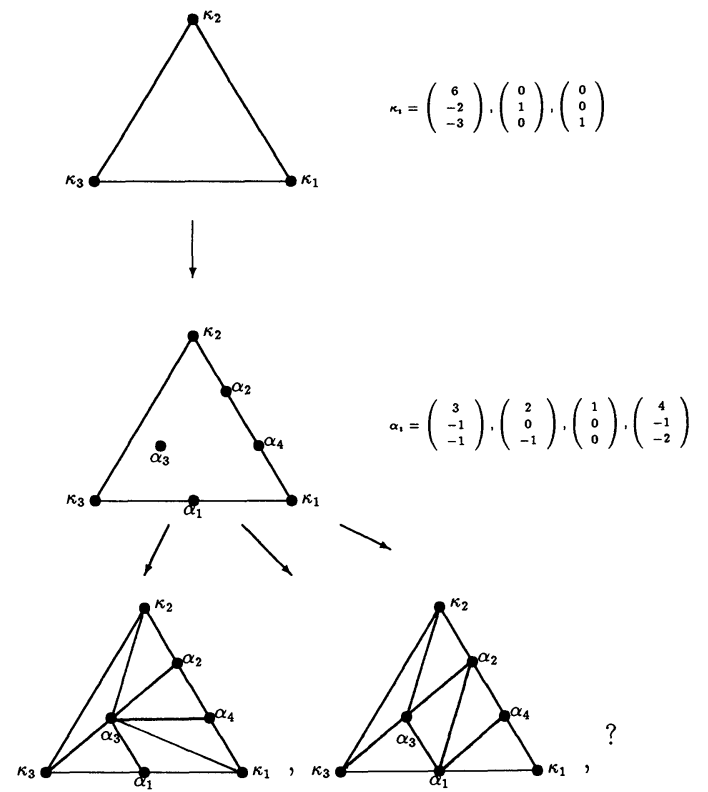

Fig. 5. Resolution of the singularities of type $\frac{1}{6}(1,2,3)$

there exist $a_{1}, a_{2} \in \mathbb{Z}$ such that

$$
a_{1} \alpha_{1}+a_{2} \alpha_{2}+\beta_{1}+\beta_{2}=0
$$

and that $C$ is then an $\left(a_{1}, a_{2}\right)$-curve.

Turning to our $A_{2} \oplus D_{4}$ orbifold, we have singularities $f=\frac{1}{6}(1,2,3)$ and we now apply the above techniques to obtain Fig. 5, i.e., we do not have a unique resolution. The reason for this can be traced to the rational curves. Whenever we have a convex quadrilateral of points in our diagram, we have two ways of dividing this into triangles. From (45) we have that convex quadrilaterals only occur around $(-1,-1)$-curves. This ambiguity is well-known in the subject of geometry of 3-folds and the process of moving from one manifold to another by using the rational $(-1,-1)$-curves is known as an elementary transformation. Figure 6 shows more on this subject. When no $(-1,-1)$-curves are present, the subdivision and hence toric resolution are unique, e.g., for the $\mathbb{Z}_{7}$-orbifold.

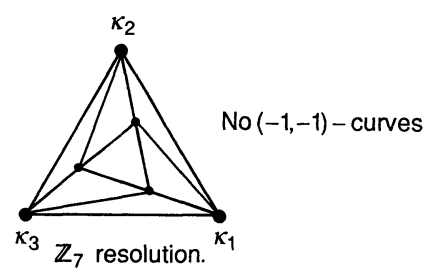

The only toric resolution of the $\mathbb{Z}_{7}$-quotient singularity from $f=\frac{1}{7}(1,2,4)$ is given uniquely by the above diagram. (This actually leads to an exceptional divisor whose irreducible components are three Hirzebruch surfaces $\Sigma_{2}$ touching at a 


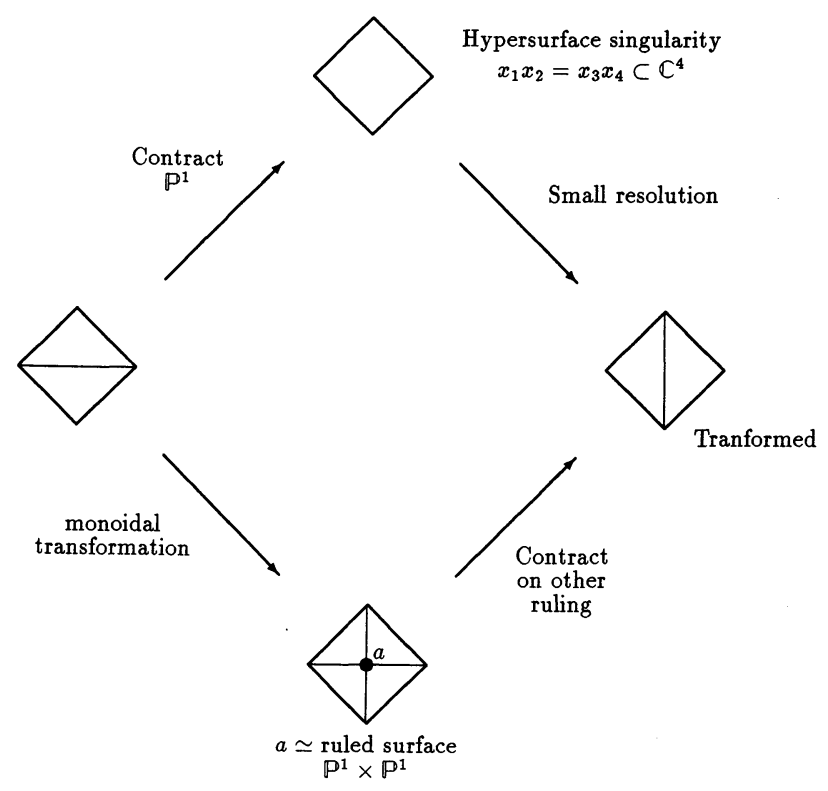

Fig. 6. Elementary transformation on a $(-1,-1)$-curve

point, where $\Sigma_{2}$ is given by the compactification of $\mathcal{O}_{\mathbb{P}^{1}}(-2)$ to give $\Sigma_{2}$ as a bundle over $\mathbb{P}^{1}$ with fibre $\mathbb{P}^{1}$.) In such cases as the $Z$-orbifold, it may be shown that the unique toric resolution is the only resolution of any kind. ${ }^{1}$

We must still check however that our resolutions are projective. We saw earlier that this may be verified for a simple blow-up giving exceptional divisor $\mathbb{P}^{n}$. We should now try and extend this reasoning to the more complicated examples here. Our argument, when constructing the very ample line bundle, depended upon the fact that the exceptional divisor $\pi^{-1}\{0\}$ had co-dimension one and had its own positive (1,1)-form (i.e., a Kähler form). In the language of toric geometry, the appearance of a manifold of co-dimension one in a blow-up corresponds to the appearance of a one-dimensional cone in the fan. The blow-up yielding this exceptional divisor corresponds to the star subdivision of the fan - namely adding the cone $\mathbb{R}_{+} n_{d}$ to our fan and then joining this cone to all neighbouring onedimensional cones. The resolution of the $\mathbb{Z}_{3}$-quotient singularity in Fig. 4 is a clear illustration of this. The so-called small resolution in Fig. 6 is an example of a resolution with no exceptional divisor since only two-dimensional cones are added to our fan.

From the above reasoning we will obtain a projective resolution if we can "factorize" the resolution into blow-ups. At each blow-up, we pull-back the Kähler form from the previous manifold and mix it in with the cohomology class associated to our exceptional divisor to obtain a new Kähler form. We eventually obtain a smooth, projective manifold if our resolution can be factorized into such blow-ups. An example concerning compact toric varieties is known [17] where we

\footnotetext{
${ }^{1}$ I wish to thank Miles Reid for pointing this out to me
} 
have a compact manifold birational to $\mathbb{P}^{3}$ which is not projective. It is easy to see from the fan representing this manifold that a series of blow-ups cannot give this space from $\mathbb{P}^{3}$. This is shown in Fig. 7 .

In our orbifold we have four cones $\alpha_{1}, \ldots, \alpha_{4}$ around which we may perform star subdivisions to give a smooth fan. We can therefore see that the ambiguity in the resolution may be thought of as due to the order in which we blow-up different parts of the total exceptional divisor. Blowing up $\alpha_{3}$ last gives a $\mathbb{P}^{2}$ component to the exceptional divisor. Blowing up $\alpha_{1}, \alpha_{2}$ or $\alpha_{4}$ last associates these to $\mathbb{P}^{1} \times \mathbb{C}$. If these are blown up earlier then the later blow-ups can perform birational transformations on the components of the exceptional divisor already present and thus if $\alpha_{3}$ is blown up first our exceptional divisor contains a component birational to, but not isomorphic to $\mathbb{P}^{2}$. We have some resolutions which cannot be factorized in the above way and may not therefore admit a Kähler metric. Figure 8 shows some examples.
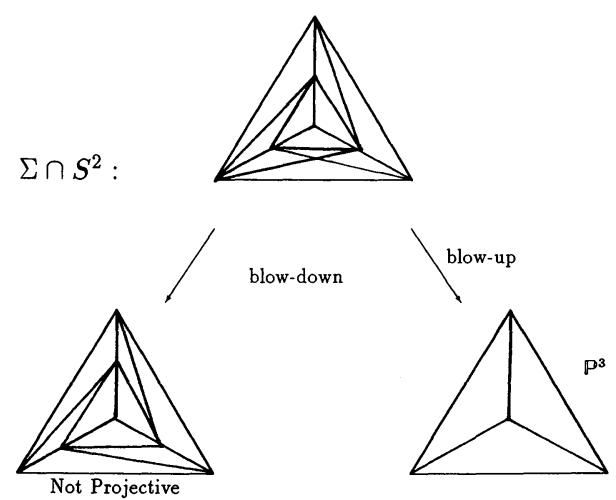

Fig. 7. A non-projective toric variety. Note this manifold is not a $\mathrm{C}-\mathrm{Y}$ and we have thus drawn the intersection of the fan with $S^{2}$
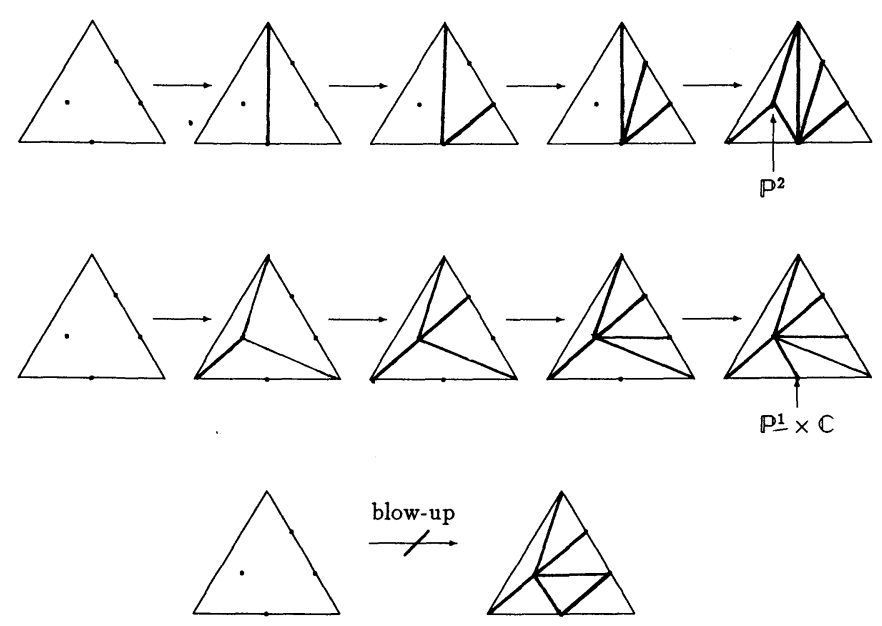

Fig. 8. Examples of different resolutions 
The resolution of the fixed tori on Fig. 1 come as light relief and it is easy to show that singularities of the form $\frac{1}{2}(1,0,1)$ blow-up to give an exceptional divisor of the form $\mathbb{P}^{1} \times \mathbb{C}$ and those of $\frac{1}{3}(1,2,0)$ give $\left(\mathbb{P}^{1} \vee \mathbb{P}^{1}\right) \times \mathbb{C}(\vee$ denotes a "plumbproduct" - i.e., joined at a point). These exceptional divisors must also "grow out" of the divisor to the $\frac{1}{6}(1,2,3)$ singularity since lines of these singularities grow out of a $\frac{1}{6}(1,2,3)$ singularity.

Looking back at Fig. 1 we can now build the total resolution of the orbifold. For the tori (A) we have four exceptional divisors of the form $\mathbb{P}^{1} \times$ torus. For the tori $(B)$ we have $3\left(\mathbb{P}^{1} \vee \mathbb{P}^{1}\right) \times$ torus. The effect of these blow-ups on the dimension of the cohomology groups is easy to calculate. We take each irreducible component of the exceptional divisor $E_{i}$ and, for this, add 1 onto $h^{1,1}=\operatorname{dim} H_{\bar{\partial}}^{1,1}(M)$ (and thus onto $h^{2,2}$ too) and add $h^{1,0}\left(E_{i}\right)$ onto $h^{1,2}$ (and thus $h^{2,1}$ ). This may be shown schematically as acting on the Hodge diamond by the following

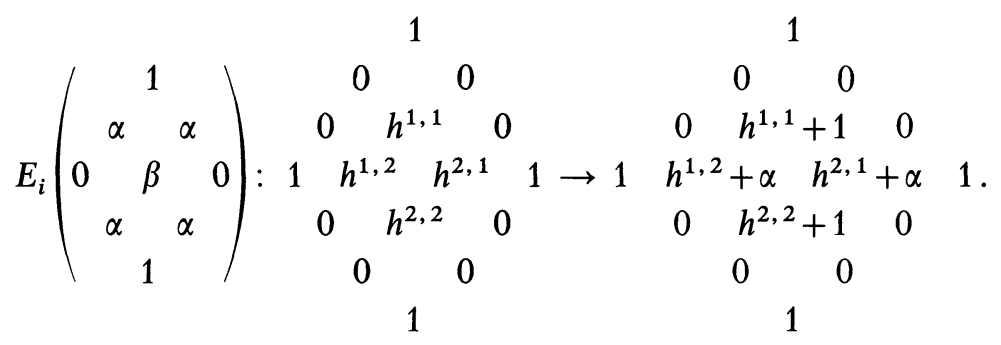

Thus (A) and (B) add 10 onto both $h^{1,1}$ and $h^{1,2}$. The (B) tori give exceptional divisors $\mathbb{P}^{1} \times$ torus from the $\mathbb{Z}_{2} \subset \mathbb{Z}_{6}$ quotient. Modding these divisors by the remaining $\mathbb{Z}_{3}$ group kills the deformation of the complex structure on the torus. The same thing happens for the $(C)$ tori. These components thus add 10 onto $h^{1,1}$ but nothing onto $h^{1,2}$. Lastly, the twelve fixed points each have 4-irreducible components to the exceptional divisor $\left(\alpha_{1}, \ldots, \alpha_{4}\right)$ but actually 3 of these are associated to the tori (B) and (C) and have thus already been counted. Each fixed point then contributes 1 to $h^{1,1}$. Starting with the six-dimensional torus we can then calculate the Hodge diamond of the smooth Calabi-Yau manifold obtained from resolving the orbifold singularities,

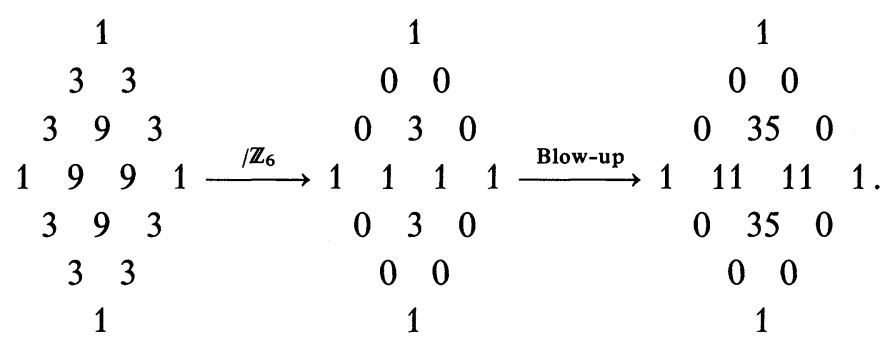

The reader should note the striking similarity between the way we constructed these numbers using twisted string ideas and using geometrical ideas.

\section{Moduli Spaces}

We should now try and construct the moduli space of the theory in the neighbourhood of our original orbifold. The conformal field theory has a set of 27 
and $\overline{27}$ representations of $E_{6}$ and so we can associate these each with a truly marginal operator. This situation can be compared to the $\hat{c}=1$ superconformal field theory [7] in which we have everywhere one dimension $\left(\frac{1}{2}, \frac{1}{2}\right)$ operator associated to each marginal operator by supersymmetry in much the same way we have constructed marginal operators from 27 and $\overline{27} \mathrm{~s}$. At certain values of radii however, we obtain an enhanced world-sheet supersymmetry allowing more marginal dimension $(1,1)$ operators to appear. We are considering an orbifold of generic radius however and we should not therefore expect any extra world-sheet supersymmetry to add to the $(2,2)$-supersymmetry we have assumed. If this is the case, our moduli space is locally simply flat space of dimension given by the number of marginal operators $=h^{1,2}+h^{1,1}=46$.

The moduli space of Calabi-Yau models at the orbifold point is quite different however. The blow-ups all give moduli space of dimension 46 but they are different sectors of space related to each other by elementary transformations. These sectors are plumbed together at the point corresponding to the orbifold to form a space which is not a manifold. Actually the situation could be even more complicated. We can contract the $(-1,-1)$-curve in the exceptional divisor to form a node locally isomorphic to the space $\left\{(x, y, z, t) \in \mathbb{C}^{4}: x y=z t\right\}$. This node can now be deformed birationally in a way described in [18] to give a smooth complex manifold with different Euler characteristic to the original blow-up and the number of moduli would also be different. This manifold can also be thought of as possible resolution of the quotient singularity. It is not necessarily true however that the resulting manifold admits a Kähler metric. If it were Kähler then our point in moduli space at which the orbifold lives would be the vertex of many cones of different numbers of dimensions.

How then can we resolve the discrepancy between the two approaches to string theory? Firstly, the above resolutions not given by blow-ups are probably not Kähler and so are illegal regions of moduli space for the theory to go into. We proved however that the many different blow-ups were Kähler and should therefore all be candidates for compactifications. It is known however [12] that as far as physics is concerned, a total explicit specification of the manifold on which we are going to compactify is more than we need. For example, a non-Ricci-flat metric can be just as good as the natural Ricci-flat metric. Another good example of this kind of equivalence is the circle and orbifold equivalence at the multicritical point of $c=1$ conformal field theories. Since all our good candidate blow-ups have the same number of marginal operators there is the possibility that they are the same conformal field theories. If they are not, we would have examples of CalabiYau manifolds which are not valid superstring compactifications.

A conformal field theory is given by its spectrum of primary fields and the three-point functions of these fields. We have already seen that the spectrum of massless states from the conformal field theory agrees with all the Calabi-Yau candidates we know admit a Kähler metric. The Calabi-Yau theory of compactification allows one, in the case of $27 \mathrm{~s}$, to calculate three-point interactions from the intersection products of homology cycles represented by surfaces in the CalabiYau [19]. These products can be calculated from the fan associated to the toric variety [20]. The different blow-ups lead to different intersection matrices for the irreducible components of the exceptional divisors and so one might draw the 
conclusion that these must therefore be physically different theories. When there is more than one irreducible component to an exceptional divisor however, it is not apparent that one identifies a certain twisted string with a certain irreducible component of an exceptional divisor. We just know that the twisted 27 strings should in some way form a basis for $H^{2}(U)$, where $U$ is the neighbourhood of the blow-up. We are therefore at liberty to consider intersection matrices $M_{i j k}, M_{i j k}^{\prime}$ equivalent if we can find $A_{i j}$ such that

$$
M_{i j k}^{\prime}=A_{i l} A_{j m} A_{k n} M_{l m n} .
$$

Actually there is no solution for $A_{i j}$ if we take the case of elementary transformations around one fixed $\frac{1}{6}(1,2,3)$-point on $M_{i j k}$ to obtain $M_{i j k}^{\prime}$. (This is because any attempt at reparameterizing $H^{2}(M)$ around one $\frac{1}{6}(1,2,3)$-resolution necessarily affects the intersections in some of the other eleven $\frac{1}{6}(1,2,3)$-resolutions due to the non-localized $\mathbb{P}^{1} \times$ torus divisors connecting these points.) This fact shows that the manifolds given by these different resolutions are differentiably distinct.

These $27^{3}$ terms are also prone to instanton contributions ([22], note this paper has interchanged the roles of $27 \mathrm{~s}$ and $\overline{27} \mathrm{~s}$ ) and this causes further discrepancy between the conformal field theory which calculates these terms exactly and the Calabi-Yau approach. It is therefore still possible that the different Calabi-Yau manifolds correspond to the same conformal field theory.

\section{Conclusions}

We have shown that the Calabi-Yau theory of superstring compactifications is not totally equivalent to the more satisfactory conformal field theory approach. It appears that too much information is specified by giving the explicit complex 3-fold complete with metric. It has already been shown that theories with Ricci-flat and non-Ricci-flat metrics on the same manifold can lead to identical physics [12], but we have presented here evidence that differentiably distinct manifolds can also give isomorphic physics. If one begins with a moduli space of Calabi-Yau compactifications, it is necessary to mod out this space by a discrete group in order to obtain the moduli space (or perhaps only part of the moduli space) of $\hat{c}=6(2,2)$ superconformal field theories. This discrete group appears to have fixed points at some of the theories given by orbifold compactifications. One is thus forced into a position of asking to what extent the compactification manifold exists. Since its structure cannot be observed it should perhaps only be held as a mathematical aid to understanding the superstring theory.

Since more is known at present about the moduli space of C-Y's than of $c>1$ conformal field theories, it should be useful to consider other parts of the $\mathrm{C}-\mathrm{Y}$ moduli space. Some points of particular interest should be the points of transition between the Calabi-Yau manifolds described by Hirzebruch [23]. We know that these manifolds are Kähler and have different Euler characteristics and so should lead to a multicritical point in the moduli space of the conformal field theory. This would provide the strongest testing ground for the compactification approach to string theory. 


\section{References}

1. Candelas, P., Horowitz, G., Strominger, A., Witten, E.: Nucl. Phys. B 258, 46 (1985)

2. Dixon, L.: Some world-sheet properties of superstring compactifications, on orbifolds and otherwise. Princeton University preprint, PUTP-1074

3. See, for example, Dijkgraaf, R., Verlinde, E., Verlinde, H.: Commun. Math. Phys. 115, 649 (1988) and references therein

4. Friedan, D., Kent, A., Shenker, S., Witten, E.: In preparation

5. Gepner, D.: Phys. Lett. 199B, 380 (1987)

6. Seiberg, N.: Observations on the moduli space of superconformal field theories. IAS preprint IASSNS/HEP-87/71

7. Dixon, L., Ginsparg, P., Harvey, J.: Nucl. Phys. B 306, 470 (1988)

8. Dixon, L., Harvey, J., Vafa, C., Witten, E.: Nucl. Phys. B 261, 678 (1985); Nucl. Phys. B 274, 285 (1986)

9. Friedan, D., Martinec, E., Shenker, S.: Nucl. Phys. B 271, 93 (1986)

10. Dixon, L., Friedan, D., Martinec, E., Shenker, S.: Nucl. Phys. B 282, 13 (1987)

11. Harmidi, S., Vafa, C.: Nucl. Phys. B 279, 465 (1987)

12. Dine, M., Seiberg, N.: Nucl. Phys. B 301, 357 (1988)

13. Griffiths, P., Harris, J.: Principles of algebraic geometry. New York: Wiley 1978

14. Pinkham, H.: Séminaires sur les Singularités des Surfaces. Demazure, M., Pinkham, H., Teissier, B. (eds.). Lecture Notes in Mathematics, vol. 777. Berlin, Heidelberg, New York: Springer 1980

15. Eguchi, T., Hanson, A.: Phys. Lett. 74B, 249 (1978)

16. Oda, T.: Convex bodies and algebraic geometry. Berlin, Heidelberg, New York: Springer 1985

17. Oda, T.: Lectures on torus embeddings and applications. Berlin, Heidelberg, New York: Springer 1978

18. Reid, M.: Math. Ann. 278, 329 (1987)

19. Strominger, A.: Topology of superstring compactification. Unified string theories. Green, M., Gross, D. (eds.). Singapore: World Scientific 1986

20. Markushevich, D., Olshanetsky, M., Perelomov, A.: Commun. Math. Phys. 111, 247 (1987)

21. Roan, S.-S., Yau, S.-T.: On Ricci-flat 3-fold. UC San Diego preprint 1986

22. Distler, J., Greene, B.: Some exact results on the superpotential from Calabi-Yau compactification. Harvard preprint HUTP-88/A020

23. Hirzebruch, F.: Some examples of threefolds with trivial canonical bundle. Lecture notes, Max-Planck-Institut für Mathematik, Bonn

Communicated by S.-T. Yau

Received November 18, 1988; in revised form October 2, 1989 
